\title{
Use of the Continuity Equation for Electromagnetic Energy Flow to Describe Radiation Transport
}

\author{
B. Ritchie
}

February 5, 1999

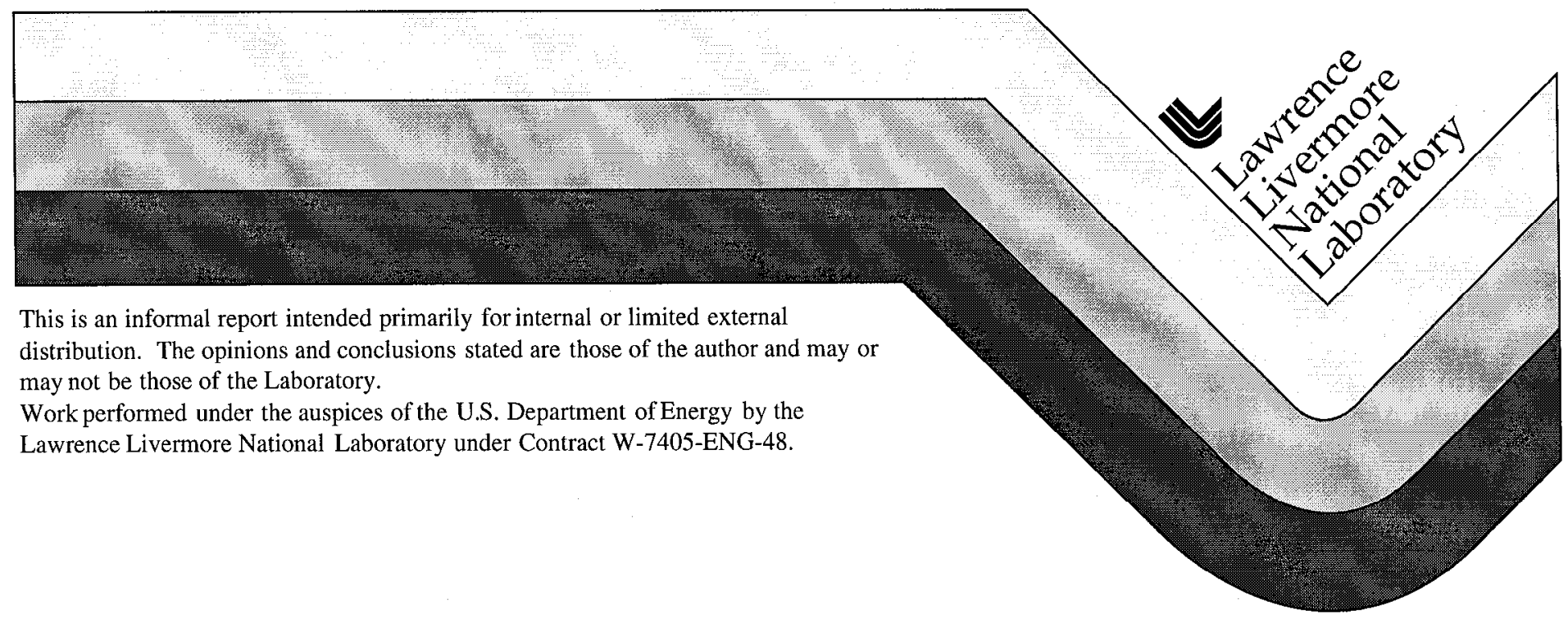




\section{DISCLAIMER}

This document was prepared as an account of work sponsored by an agency of the United States Government. Neither the United States Government nor the University of California nor any of their employees, makes any warranty, express or implied, or assumes any legal liability or responsibility for the accuracy, completeness, or usefulness of any information, apparatus, product, or process disclosed, or represents that its use would not infringe privately owned rights. Reference herein to any specific commercial product, process, or service by trade name, trademark, manufacturer, or otherwise, does not necessarily constitute or imply its endorsement, recommendation, or favoring by the United States Government or the University of California. The views and opinions of authors expressed herein do not necessarily state or reflect those of the United States Government or the University of California, and shall not be used for advertising or product endorsement purposes.

This report has been reproduced directly from the best available copy.

Available to DOE and DOE contractors from the

Office of Scientific and Technical Information

P.O. Box 62, Oak Ridge, TN 37831

Prices available from (423) 576-8401

Available to the public from the

National Technical Information Service

U.S. Department of Commerce

5285 Port Royal Rd.,

Springfield, VA 22161 
Use of the continuity equation for electromagnetic energy flow to describe radiation transport

\section{Burke Ritchie}

University of California

Lawrence Livermore Narional Laboratory

Livermore, California 94550

The continuity equation for electromagnetic (EM) energy

flow is used to describe radiation flow in the transport regime. The geometric-optics limit of the EM continuity equation is the transport equation, which depends on the time $t$, a position variable $\vec{r}$, and parametrically on a unit vector in the ray direction $\hat{k}$, making it an extremely difficult equation to implement computationally. The EM continuity equation however depends only on $t$ and $\vec{r}$. An average with respect to a manageable number of EM field polarization directions, which is needed to describe the flow of unpolarized radiant energy, gives an energy density which is continuousy distributed in $\widehat{k}$, thereby eliminating "ray effect" distortion, which occurs because the transport equation, for computational reasons, is discretely distributed in $\widehat{\mathrm{k}}$. 
In numerous applications radiation transport through a material is dominated by emission, absorption, and re-emission, while scattering is down by many orders of magnitude and can be ignored. The transport equation in such regimes is [1],

$$
\frac{1}{\mathrm{c}} \frac{\partial \mathrm{I}}{\partial \mathrm{t}}+\hat{\mathrm{k}} \cdot \vec{\nabla} \mathrm{I}=-\kappa_{\mathrm{a}} \mathrm{I}+\mathrm{S}
$$

where $\mathrm{I}$ is an energy density, $\widehat{k}$ is a unit vector in the ray direction, $\kappa_{\mathrm{a}}$ is an absorptive opacity, and $\mathrm{S}$ is a suitably normalized emissive source isotropically distributed with repect to $\widehat{\mathrm{k}}$. Although deceptively simple in appearance Eq. (1) is extremely difficult to implement computationally because of its parametric dependence on the ray direction $\hat{k}$. Formal solutions are of little use because of the nonlinear dependence of the source $S$ on the energy density 1 through the material temperature. Thus in practical applications the equation must be solved numerically on discrete grids in time and space, as well as in $\widehat{k}$. (The source and opacity also depend on the magnitude of the wave vector, which is $\omega / \mathrm{c}$ for radiant energy, where $\omega$ is the frequency, which also must be discretized.)

Fig. 1 shows an example of the use of Eq. (1) to describe radiation page 2 
transport, where the principal inaccuracy is the "ray effect" or lumpy distribution in the free space region outside the region of emission due to an insufficient number of values of $\hat{\mathrm{k}}$. The details of the numerical procedures used in all of the calculations in this paper can be found elsewhere [2-3].

Obviously it should be unnecessary to resolve the problem into a set of ray directions in order to describe the physics of this situation because, in absence of scattering, there is no preferred initial or "in" set of directions for the rays. The final or "out" direction occurs, from the point of view of wave optics, as we shall see below, through finding the geometric-optics limit of Maxwell's theory of wave propagation, in which the propagation loses its dependence on $\omega / c$ and depends only on $\widehat{k}$. In this paper however we show that one can make good use of the loss of dependence on $\omega / \mathrm{c}$ by solving Maxwell's equations directly and representing a continuously distributed $\hat{\mathrm{k}}$ implicitly and automatically through the transverse dependence of $\widehat{k}$ on the field polarization directions, which is guaranteed by Maxwell's equations. In the problems of 
interest of course the radiation is not polarized; thus it is necessary and, from the satisfactory nature of our results, sufficient to average the energy density over all possible polarization directions of the Maxwell fields to describe the flow of unpolarized radiant energy. Hence the "lunch" is not free. The discretization of the problem is now in time and space and in the polarization directions of the Maxwell fields; however it turns out that the polarization average can be achieved with a fairly modest number of discrete polarization directions. Furthermore, since $\widehat{k}$ is never discretized the ray effect distortion disappears (Fig. 2). Finally we recall that it is only the independence of the propagation on $\omega / c$ which permits us to solve Maxwell's equations at all since otherwise one would have to resolve the fields on the scale of $\omega / c$, typically in the regime of short wavelengths, such that it would be impossible in the problems of interest to describe the transport of radiation over millions or billions of these wavelengths. In other words the continuity equation for electromagnetic (EM) energy flow can be used to simulate the average energy density directly - the energy density of Eq. (1) integrated over the solid angle defined by $\mathrm{d} \hat{\mathrm{k}}$ and page 4 
divided by $4 \pi$ - as shown in Fig. 2 , without first resolving the motion into a set of $\hat{k}$ directions.

The equations for our model [4-6] are,

$$
\begin{gathered}
\frac{1}{\mathrm{c}} \frac{\partial \mathrm{u}}{\partial \mathrm{t}}+\vec{\nabla} \cdot(\overrightarrow{\mathrm{E}} \times \overrightarrow{\mathrm{H}})=-\kappa_{\mathrm{a}} \mathrm{u}+\mathrm{S} \\
\frac{1}{\mathrm{c}} \frac{\partial \overrightarrow{\mathrm{E}}}{\partial \mathrm{t}}+\kappa_{\mathrm{a}} \overrightarrow{\mathrm{E}}=\vec{\nabla} \times \overrightarrow{\mathrm{H}}
\end{gathered}
$$

Maxwell's equations for the electric, magnetic fields $\overrightarrow{\mathrm{E}}, \overrightarrow{\mathrm{H}}$ have been manipulated in the usual way [4] to arrive at a continuity equation for the energy density $u=1 / 2\left(E^{2}+H^{2}\right)$. This form is desirable because then we can then sensibly introduce a phenomenological emissive source $S$, which has no counterpart in the equations for the fields themselves. Eq. (2a) is the result. The model defined by Eqs. (2) does not use Maxwell's equation for the magnetic field,

$$
\vec{\nabla} \times \overrightarrow{\mathrm{E}}=-\frac{1}{\mathrm{c}} \frac{\partial \overrightarrow{\mathrm{H}}}{\partial \mathrm{t}},
$$

other than to derive Eq. (2a) without S. The model does use Maxwell's equation for the electric field [Eq. (2b)] because it describes the absorption of the electric field in the material [5-6]. We infer the magnetic field for use in Eqs. (2) from the energy 
density $u$ in a way which we describe below.

First however we briefly review how Eq. (2a) reduces to Eq. (1) in the geometric-optics limit of Maxwell's equations [7]. One uses the Fourier representations for the fields,

$$
\begin{aligned}
& \overrightarrow{\mathrm{E}}=\vec{\varepsilon} \mathrm{e}^{\mathrm{i}\left(\overrightarrow{\left.k_{k r} \vec{r}-\omega t\right)}+c c\right.} \\
& \vec{H}=\vec{h} \mathrm{e}^{\mathrm{i}(\vec{k} \overrightarrow{c o r}-\omega t)}+c c .
\end{aligned}
$$

From Eq. (3) we infer that,

$$
\widehat{\mathrm{k}} \times \vec{\varepsilon}=\overrightarrow{\mathrm{h}},
$$

where the envelopes of the fields in Eqs. (4) are assumed to be slowly varying in space and time on the scale of $(\omega / c)^{-1}$ and $\omega^{-1}$ respectively. Using Eqs. (4) and Eq. (5) (and its complex congugate) in Eq. (2a) and the vector identities,

$$
\begin{aligned}
& \quad \vec{\varepsilon}^{*} \times \hat{\mathrm{k}} \times \vec{\varepsilon} \cong \hat{\mathrm{k}} \cdot \vec{\varepsilon}^{*} \cdot \vec{\varepsilon} \\
& \left(\hat{\mathrm{k}} \times \vec{\varepsilon}^{*}\right) \cdot(\hat{\mathrm{k}} \times \vec{\varepsilon}) \cong \vec{\varepsilon}^{*} \vec{\varepsilon} \\
& \text { where the term } \overrightarrow{\mathrm{k}} \cdot \vec{\varepsilon} \text { and its complex conjugate are negligible on the }
\end{aligned}
$$
page 6 
slow scale [7] since in absence of free charge $\vec{\nabla} \cdot \vec{D}=0$, where $\vec{D}=\vec{E}+4 \pi \vec{P}$ is the displacement field due to the material polarization field $\overrightarrow{\mathrm{P}}[5-6]$,

$$
\vec{P}=\frac{i \kappa_{a} c}{4 \pi \omega} \vec{\varepsilon} e^{i(\vec{k} \cdot \vec{r}-\omega t)}+c c
$$

These are the standard results for the geometric-optics limit [7]. Substituting Eqs. (4) into Eq. (2a) and using Eqs. (5) and (6) and their complex conjugates, one arrives at Eq. (1), where the energy density is interpreted as,

$$
I=\vec{\varepsilon} \cdot \vec{\varepsilon}+c c
$$

Thus the transport equation, which in fact is just a conservation of mass equation which applies generically to particle transport, is consistent with the geometric-optics limit of Maxwell's wave theory, in which the propagation is described as the straight-line transport of a light ray, whose intensity is given by Eq. (8), in the direction of $\hat{\mathrm{k}}$.

Now let us solve Eqs. (2) in this limit directly, without using the substitution of Eqs. (4). At first glance this may appear to be 
impossible because, according to Eqs. (4), the fields themselves, but not their envelopes, are rapidly varying in space and time. However in a sense Eqs. (4) are misleading because the rapidly varying carrier waves contain more information, namely the wavelength $2 \pi(\omega / c)^{-1}$, than we need to describe the problem posed phenomenologically by the emissive source in Eq. (2a), S, which is just a radiant energy distribution, usually modelled using the Planckian function [1], which exists on the slow scale. From this point of view Eqs. (2) are already coarse-grained. Indeed the slow-scale equation, namely the transport equation given by Eq. (1), also contains an excess of information, namely the "light ray" direction $\widehat{k}$, than posed by the phenomenological source, in agreement with our original surmise that this level of resolution is excessive to describe a physical situation in which there is no initial set of preferred directions, either from the source or from "in" scattering. Thus within such a restricted situation, which however includes numerous practical applications, Eqs. (2) seem physically appropriate to describe the motion or flow of radiant energy in the so-called transport regime. 
The model given by Eqs. (2) is implemented as follows. The source in Eq. (2a), $S=\kappa_{a} B$, where $B$ is the Planckian function [1], turns on at initial time allowing the average energy density, $u$, to evolve from an initial zero value over an increment of time $\mathrm{dt}$. In this model the advection of the radiation [second term on the left side of Eq. (1)] is described by the divergence of the electromagnetic (EM) flux divided by $c$ [second term on the left side of Eq. (2a)]. This effect and the absorption are ignored during the first temporal increment and are calculated for each subsequent increment using the information from the previous time step. The electric and magnetic fields needed to calculate the EM flux are calculated as follows. Since Eq. (2a) is an equation for the temporal evolution of the electric field, the magnetic field is inferred from a knowledge of $u=H^{2}$, where we have used Eq. (5) and Eq. (6b) to infer that $H^{2}$ is sensibly equal to $E^{2}$ in our regime. The three components of the magnetic field are calculated from the square root of $u$, where the choice of their amplitudes will be described shortly. Finally these values of the magnetic field are substituted into the right side of Eq. (2b), which is solved for the E-field components. In the next 
temporal increment Eq. (2a) is advanced and the information calculated from the previous time step is used to calculate the divergence of the EM flux divided by $\mathrm{c}$ and the opacity term. In optically thick problems it is necessary to use an implicit advancement algorithm for the opacity terms in Eqs. (2). Such an algorithm can be constructed using the methods described previously [2].

The amplitudes of the $\mathrm{H}$-field components are chosen as follows. In the 2D problem considered here (Figs. 1-3) the motion is in the $x-y$ plane. EM transversality means that unpolarized energy flow in this plane can be simulated by picking a series of $\mathrm{H}$ components weighted from zero to one on the positive axis perpendicular to the plane (or by symmetry from zero to minus one on the negative perpendicular axis). The amplitudes of the in-plane $H$ components are then chosen at every time step from a random number generator in the range of values from minus one to plus one. The three amplitudes of the vector field are then normalized so that the sum of their squares is one, in order to satisfy the magnitude condition $H_{x}{ }^{2}+H_{y}{ }^{2}+H_{z}{ }^{2}=u$. 
The result is shown in Fig. 2, which is an average over twentyone $\mathrm{H}_{\mathrm{z}}$ polarization amplitudes from zero to one. The average over eleven amplitudes gives a result which agrees with the result of Fig. 2 to better than $1 \%$. Thus the method converges rapidly for a modest number of discrete amplitudes; it is necessary only that this number spans the range of possible amplitudes from zero to one, subject to the normalization condition given above.

Other schemes have been tried and rejected as containing bias.

For example use of fixed rather than stochastic in-plane components $\left(H_{x}\right.$ and $\left.H_{y}\right)$ leads to results which follow fixed two-way ray directions, up and down, left and right, or diagonally to any two opposite corners. Such a scheme appeared have all of the bias of ray transport and to require an average over a large number of amplitude choices to beat down the bias. At the opposite extreme all advection vanishes if the amplitudes of all three components of $H$ are chosen using a random number generator.

In summary the scheme of a discrete representation of $\mathrm{H}_{\mathrm{z}}$ and a stochastic representation of $H_{x}, H_{y}$ gives an effectively continuous 
representation of directed energy in the $x-y$ plane, such that the ray effect of transport theory (Fig. 1) is absent (Fig. 2). Since the inplane amplitudes are generated at each time step, it is necessary to store only the perpendicular amplitudes and the results appear to be robust with respect to the coarseness of this discretization.

Evidently the generalization to three dimensions would require three such discrete sets for the three planes of the motion.

Although there are small quantitative differences in the distributions shown in Figs. 1 and 2, the total energies obtained by integrating the average energy densities over space are in close agreement, as shown in Fig. 3.

Acknowledgement. The author would like to thank Pieter Dykema, Merle Riley, John Castor, and Stephen Libby for valuable discussion on this work. 


\section{References}

1. Zeldo'vich, Ya. B. and Raizer, Yu. P., 1966, Physics of Shock Waves and High-Temperature Hydrodynamic Phenomena (Academic Press, New York), pp.128-130.

2. Ritchie, B., Dykema, P., and Braddy, D., 1997, Phys. Rev. E 56, 2217. Two typographical errors are noted: (1) $C_{v}$ in Eq. (7) should be multiplied by the material density $\rho$ in $\mathrm{g} \mathrm{cm}^{-3}$ and (2) the caption to Fig. 10 should contain nonzero rather than zero scattering opacity.

3. Ritchie, B. and Riley, M. E., 1997, Sandia Report Sand97-1205, UC401.

4. Jackson, J. D., 1975, Classical Electrodynamics, 2nd Ed. (Wiley, New York), p. 237.

5. Icsevgi, A. and Lamb, W. E., 1969, Phys. Rev. 185, 517.

6. Garrison, J. C., Ritchie, B., Nathel, H., Hong, C. K., and Minner, L., 1991, Phys. Rev. A 43, 4941.

7. Born, Max and Wolf, Emil, 1980, Principles of Optics, 6th Ed. (Pergamon Press, Oxford), p. 110-111. 


\section{Figure Captions}

Fig. 1 Energy density from Eq. (1) averaged over the solid angle defined by $d \hat{k}$ according to the $S_{N}$ scheme for $N=8$. A square region of emission ( $\kappa_{a}=5 \mathrm{~cm}^{-1}$ and $B=1$ for a source $S=\kappa_{a} B$ ) between the coordinates $-1.0,-0.6 \mathrm{~cm}$ and $0.6,1.0 \mathrm{~cm}$ on the $\mathrm{x}$ and $\mathrm{y}$ axes encloses a region of absorption $\left(\kappa_{\mathrm{a}}=5 \mathrm{~cm}^{-1}, \mathrm{~B}=0\right)$ and is surrounded by a region of free space $\left(\kappa_{\mathrm{a}}=0, \mathrm{~B}=0\right)$. The calculation uses a $64 \times 64$ spatial grid and 1001 points in the ct dimension for a maximum ct of $2 \mathrm{~cm}$.

Fig. 2. Average energy density from Eqs. (2). Other details are the same as in Fig. 1.

Fig. 3. Total energy: energy density of Fig. 1 (dashed) and Fig. 2 (solid) integrated over all space. 


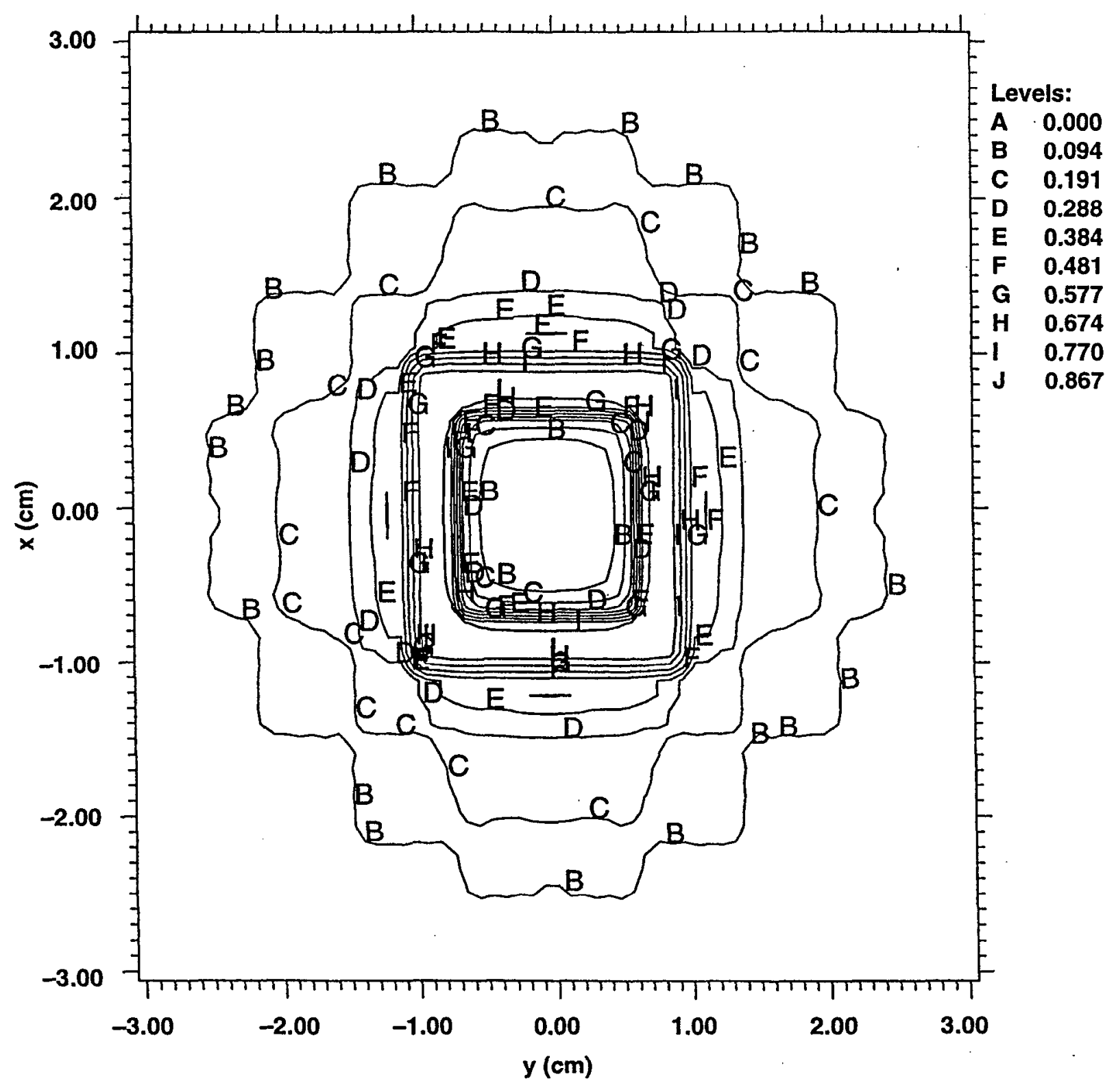




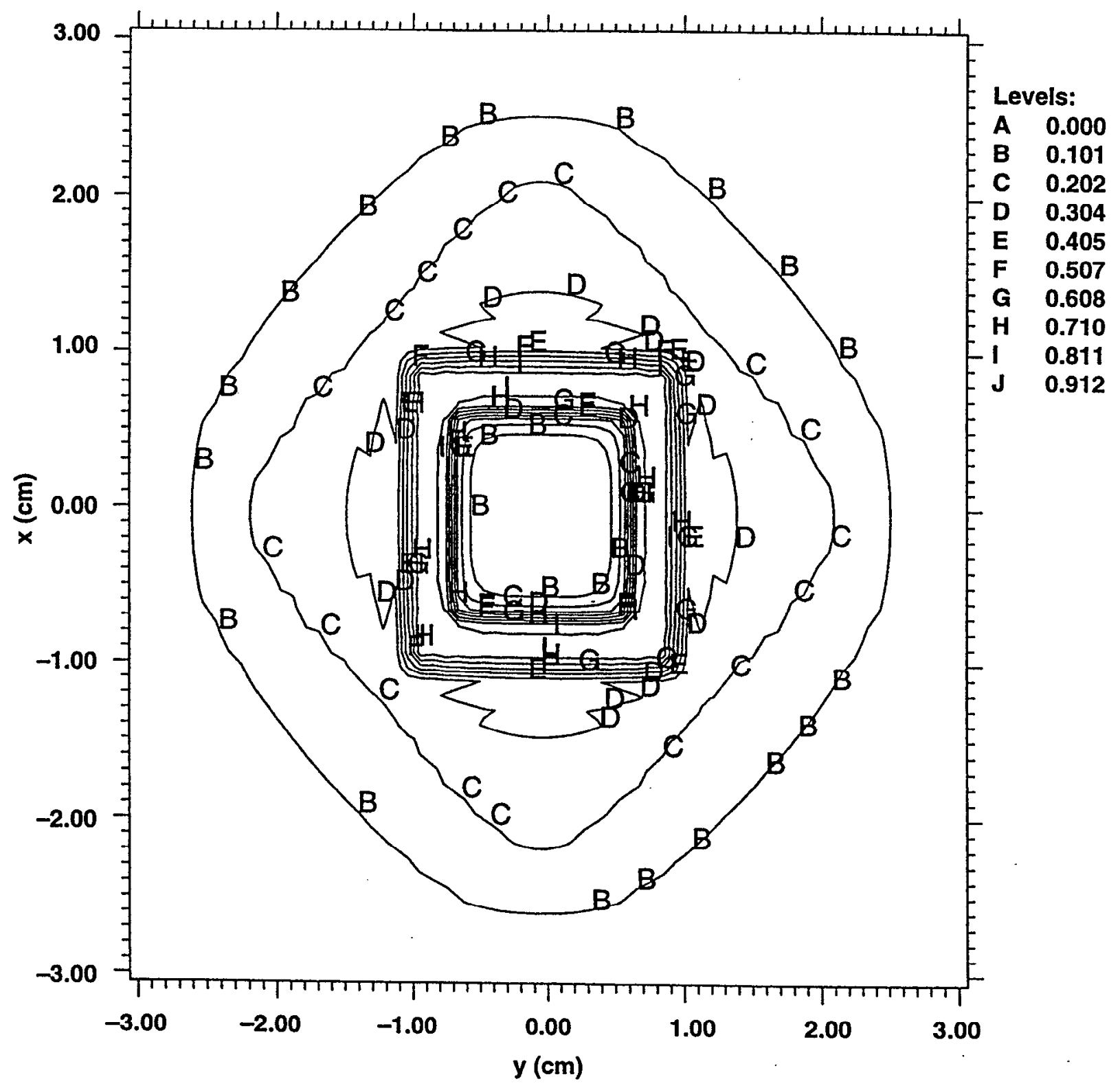




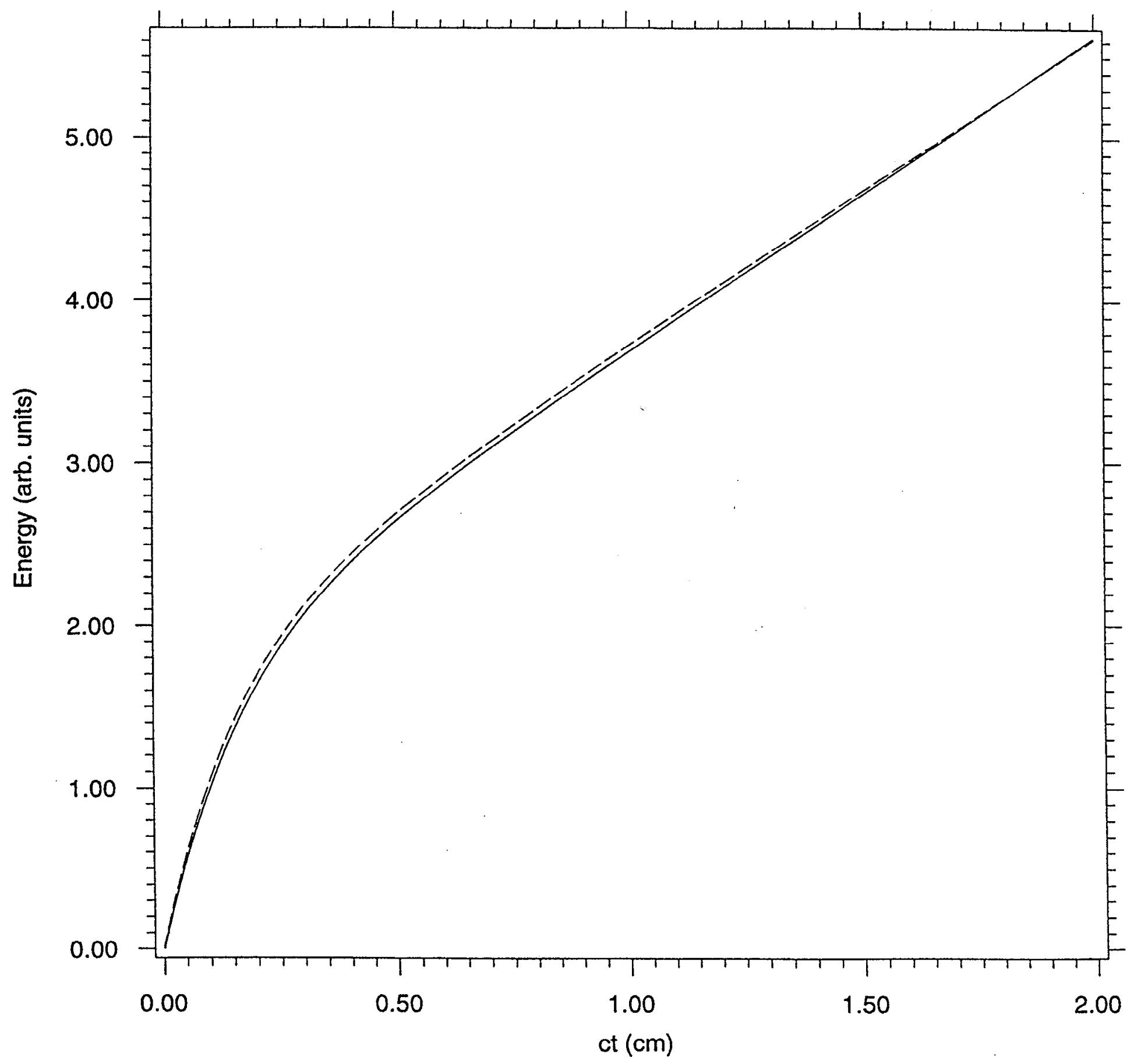

